\title{
Effect of Fixative Solutions on Non-Heme Iron Staining in the Brain
}

\author{
Mi-Rui Liu ${ }^{1}$, Yu Jin ${ }^{1}$, Ji-Feng Song, Xiao-Feng Zhang, Yan-Zhong Chang and Peng Yu* \\ Key Laboratory of Animal Physiology, Biochemistry and Molecular Biology of Hebei Province, Laboratory of Molecular Iron Metabolism, Hebei Normal \\ University, China \\ ${ }^{1}$ These two authors contribute equally to this work.
}

*Corresponding author: Peng Yu, Associate Professor, Laboratory of Molecular Iron Metabolism, College of Life Science, Hebei Normal University, Nanerhuan Eastern Road, Shijiazhuang, China

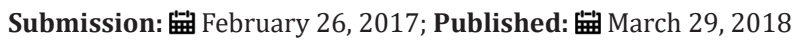

\begin{abstract}
Objective: Non-heme iron staining is very weak in the brain due to its limited iron contents. In order to optimize the iron staining in the brain, different types of fixation solutions were explored and compared in this study.

Methods: The brain tissues were cut from mice and fixed with 95\% ethanol, or a mixture of methanol: chloroform: acetic acid (volume ratio is 6:3:1), or Bouin's solution or polyformaldehyde. Then, coronal frozen sections were prepared and iron histochemistry was performed to detect the iron staining in cerebral cortex, hippocampus, cerebellum and choroid plexus. Finally, the images were captured and saved for analysis.

Results: It was found that the different types of fixation solutions didn't have obvious influences on iron staining when the incubation time was proper in Perl's staining solution.
\end{abstract}

Conclusion: Optimal iron staining could be achieved with a combination of DAB-enhancement, and fixative solution could be selected according to other considerations in experiments. This provided important data for establishing a steady method for positive iron staining in the brain tissues.

Keywords: Iron; Bouin's solution; Polyformaldehyde; Perl’s solution; Brain

\section{Introduction}

Iron is the most abundant transition metal in the body. Iron plays important roles in oxygen transport and utilization, the synthesis of neurotransmitter and acts as co-factors for many enzymes in cell metabolism and energy transduction [1,2]. Iron overload is related with neurodegenerative diseases (NDs) such as Parkinson's Disease (PD) and Alzheimer's Disease (AD) [2,3]. Moreover, brain iron deficiency in children can induce irreversible mental retardation, and iron deficiency can decrease the performance of adults [4,5]. Therefore, it is very important to keep iron homeostasis in the brain.

In recent years, iron metabolism has become more and more important for understanding the brain function. To detect the iron contents and distribution is the basis for research of brain iron metabolism. It is reported that approximately $70 \%$ of the total iron exists in the form of heme iron (ferrous protoporphyrin IX), such as in hemoglobin, myoglobin, cytochromes and neuroglobin [6]. Due to the limited non-heme iron contents in the brain, the detection of non-heme iron is not easy. Iron histochemistry such as Perl's staining is one of the traditional methods to detect non-heme iron contents in situ. This method has three main advantages in clinical and fundamental research. Firstly, iron staining is performed in sections, these series of sections can also be used for immune histochemistry, Nissl staining and TUNEL detection, which could give more data with good correspondence, help to analyze their relationships and save the number of animals in research. Secondly, the detection of non-heme iron is in situ and can be observed with microscope, which give visible and morphological data. Thirdly, this method does not need special large-scale instruments compared with other methods of iron detection, such as atomic absorption spectrophotometer (AAS), synchrotron-based micro$\mathrm{X}$-ray fluorescence $(\mu$-XRF) and inductively coupled plasma mass spectrometer (ICP-MS) [7]. Non-heme iron staining can accurately reflect the iron contents in situ, and has been widely used for iron staining in peripheral tissue sections. Ferric iron in tissues is not combined tightly enough, and can be released after incubation with dilute hydrochloric acid. After that, released ferric iron can bind ferrocyanide called Prussian blue reaction, which gives a brilliant blue mixed-valence iron (II/III) complex observable under light microscope [8-11]. However, iron contents in the brain are much less than peripheral tissues such as liver, spleen and so on, and the product of Prussian blue reaction is not blue under physiological conditions [12]. There are some factors influencing the staining of non-heme iron in the brain, fixative solution was one of them, 
so different fixative solutions were used and compared in this study, which gave some suggestions for the optimal non-heme iron staining in the brain.

\section{Materials and Methods}

\section{Chemicals and reagents}

APES and 3, 3'-diaminobenzidine (DAB) were from ZSGB-Bio (Beijing, PRC). Bouin's fixative solution was from Solarbio Life Sciences (Beijing, PRC). Paraformaldehyde, methanol, chloroform, acetic acid, potassium ferrocyanide, concentrated hydrochloric acid, pentobarbital sodium, hydrogen peroxide and other chemicals and regents were purchased from YONGDA Chemical Reagent Co. Ltd. (Tianjin, PRC).The water used in the experiments is de-ionized water(Resistance value $\geq 18.2 \mathrm{M} \Omega$ )

\section{Animals and treatments}

Male Balb/c mice which weighed $30 \pm 5 \mathrm{~g}$ were purchased from HEB LAC in Hebei Medical University (Hebei Province, PRC), and they were divided into 4 groups, each group contained 3 mice. After mice were anesthetized with pentobarbital sodium (40mg/ $\mathrm{kg}$, i.p.), they were per-fused through the ascending aorta for a minimum of $5 \mathrm{~min}$ with ice-cold normal saline $(0.9 \% \mathrm{NaCl})$, then the brain was cut and fixed respectively in $95 \%$ ethanol, a mixture of methanol: chloroform: acetic acid (volume ratio is 6:3:1, mixture group), Bouin's solution and 4\% poly-formaldehyde. All animal experiments were performed in accordance with regulations for the administration of affairs concerning experimental animals (1997.539) by the State Science and Technology Commission of People's Republic of China.

Results

\section{Preparation of brain sections}

Fixative solutions were removed and the brain tissues were rinsed in $0.1 \mathrm{M}$ PB ( $\mathrm{pH}$ 7.2-7.4). Next, mouse brains were dipped into $30 \%$ sucrose in $0.1 \mathrm{M}$ PB for 2 days. Then brain sections were cut coronally at $20 \mu \mathrm{m}$ thickness on a freezing microtome, and attached to APES-coated glass slides, as previously described [14]. Iron is accumulated in the spleen of ceruloplasmin gene knockout (CP-/-) mice, so spleen sections from CP-/- mice were used as the positive control.

\section{Iron histochemistry}

Non-heme iron was stained with DAB-enhanced iron histochemistry method as previously described [13,14]. After rinsing three times in de-ionized water for $30 \mathrm{~min}$, the sections were incubated at room temperature for $20 \mathrm{~min}$ in $1 \% \mathrm{H}_{2} \mathrm{O}_{2}$ in $0.01 \mathrm{M}$ phosphate-buffered saline (PBS) to quench endogenous peroxidase activity and then rinsed three times in de-ionized water for $30 \mathrm{~min}$. After that, they were incubated at room temperature in a freshly made Perl's solution [ $2 \%$ potassium ferrocyanide in $2 \%$ aqueous hydrochloric acid] for $1 \mathrm{~h}$ and $8 \mathrm{~h}$ respectively, and subsequently rinsed three times (10min each) in de-ionized water and the staining was enhanced in a diaminobenzidine (DAB) solution. Negative control sections were incubated in Perl's solution, but with PBS in place of potassium ferrocyanide. The control slides did not show any positive staining. Finally, the sections containing midbrain, cerebellum, cerebral cortex, $\mathrm{CPu}$, hippocampus and choroid plexus were selected to show the non-heme iron staining, and they were photographed with a Zeiss Imager A2 microscope (Jena, Germany).

\section{Non-heme iron staining needs to be enhanced by DAB in the brain}

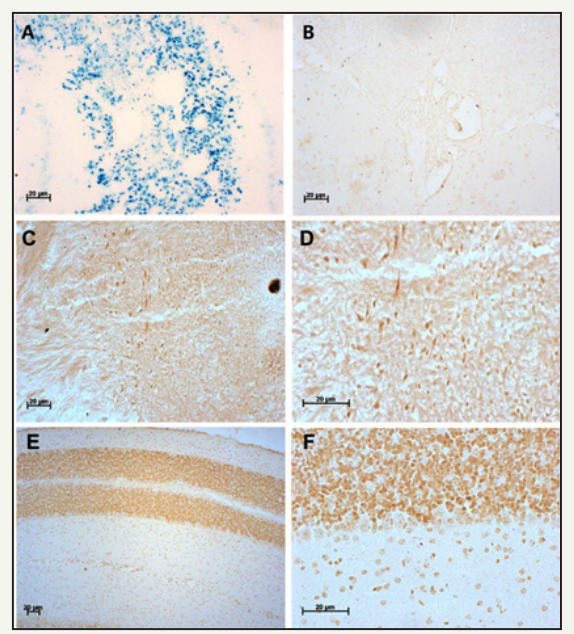

Figure 1: Non-heme iron staining in spleen and brain.

Figure 1A: Spleen, positive control of non-heme iron staining without DAB enhancement.

Figure 1B: Choroid plexus in the brain, negative control of non-heme iron staining with DAB enhancement.

Figure 1C \& Figure 1D: Midbrain of non-heme iron staining with DAB enhancement after fixation with $95 \%$ ethanol.

Figure 1E \& Figure 1F: Cerebellum of of non-heme iron staining with DAB enhancement after fixation with Bouin's solution. The time of incubation with Perl's solution is 1 hour for A, and 8 hours for B-E. Scale bars: $20 \mu \mathrm{m}$. 
Non-heme iron (ferric iron) was stained in brain sections with iron histochemistry method, and spleen section with high level of iron was used as the positive control. Data showed that the staining is blue in spleen after incubation in Perl's solution for 1hour (Figure $1 \mathrm{~A})$, which indicated that the procedure in this study worked well and that spleen has so much iron that the staining presented as blue in such a short reaction time. At the same time, blue staining of non-heme iron was not observed in the brain sections. After DAB enhancement, brown staining of non-heme iron could be seen in the midbrain and cerebellum (Figure 1B), which was confirmed by the negative control sections with the same incubation and staining procedures (Figure 1C-1F).

\section{Different fixative solutions do not induce obvious changes in non-heme iron staining.}

Four kinds of different fixative solutions were detected to see their effects on DAB-enhanced non-heme iron staining, data showed that no obvious positive staining in both mixture group and poly-formaldehyde group after incubation in Perl's solution for 1 hour (Figure 2A, 2B and 2E). When the incubation time increased up to 8hours, the positive staining could be clearly seen in the midbrain (Figure 1C, 1D), cerebellum (Figure 1E, 1F), CPu (Figure 2C), cerebral cortex (Figure 2D), choroid plexus (Figure 2F, 2H) and hippocampus (Figure 2G). Moreover, there are no significant changes of brown staining among different fixative treatments of 95\% ethanol, Bouin's solution and poly-formaldehyde.

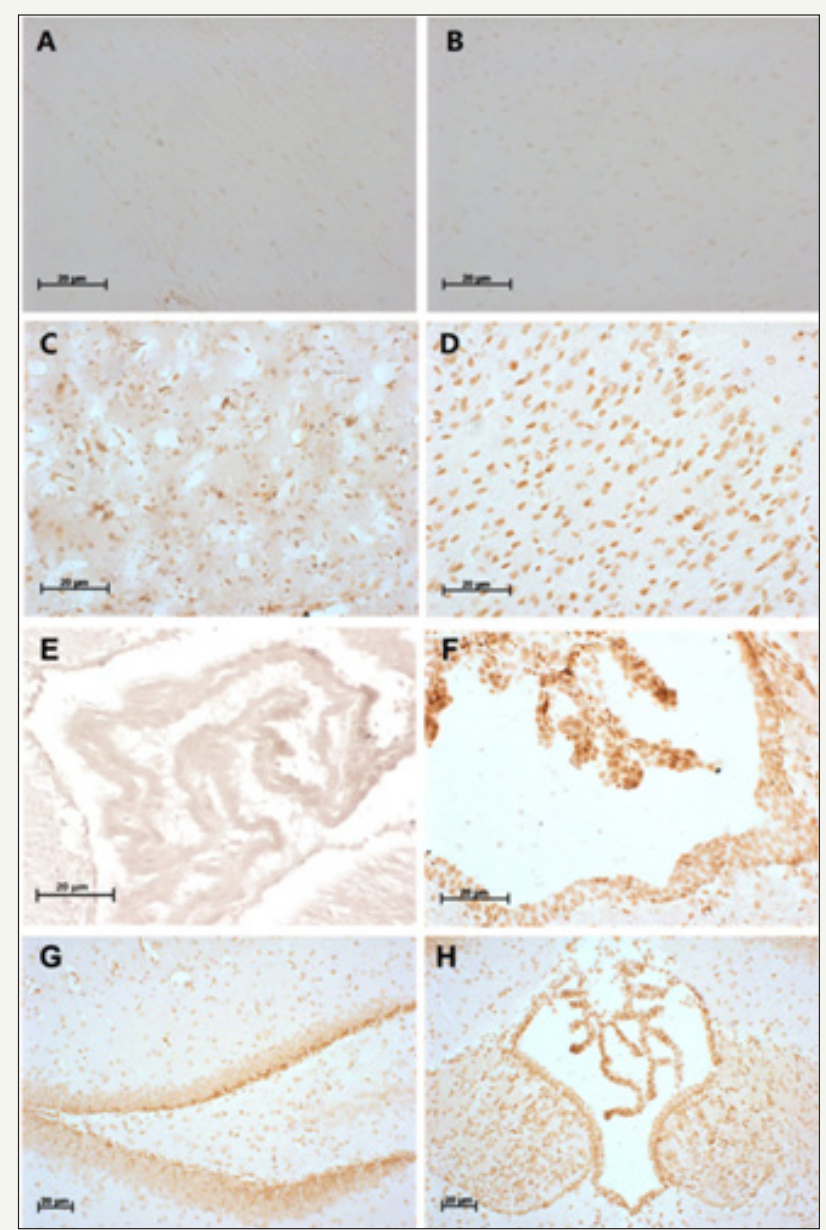

Figure 2: : Non-heme iron staining with DAB enhancement.

Figure 2A: a mixture of methanol: chloroform: acetic acid as the fixative solution, corpus collosum, incubation with Perl's solution for 1 hour.

Figure 2B: a mixture of methanol: chloroform: acetic acid as the fixative solution, cerebral cortex, Perl's solution for 1 hour.

Figure 2C: poly-formaldehyde as the fixative solution, CPu, Perl's solution for 8hours.

Figure 2D: Bouin's solution as the fixative solution, cerebral cortex, Perl's solution for 8 hours.

Figure 2E: poly-formaldehyde as the fixative solution, choroid plexus, Perl's solution for 1 hour.

Figure 2F: poly-formaldehyde as the fixative solution, choroid plexus, Perl's solution for 8 hours.

Figure 2G: Bouin's solution as the fixative solution, hippocampus, staining for 8 hours.

Figure 2H: Bouin's solution as the fixative solution, choroid plexus, Perl's solution for 8 hours. Scale bars: $20 \mu \mathrm{m}$. 


\section{Discussion}

Iron metabolism participates in so many cellular metabolism processes and iron homeostasis has gained more and more attentions in clinic and basic research [15]. Detection of iron levels is primary and necessary in research of iron metabolism. Iron histochemistry is one of the iron detection methods. It is easy to handle, and can get the observable blue color and morphological data of non-heme iron staining in situ, so it is strongly recommended for brain tissue sections especially with small sample size and complicated structures such as choroid plexus, hippocampus, $\mathrm{CPu}$ and so on.

However, non-heme iron levels are very low comparing with those of peripheral organs and tissues, which make the staining difficult and the optimal staining conditions to be investigated. In our studies, we mainly focused on the influence of different fixative solutions on the iron staining, and spleen sections were used as positive control because iron is accumulated in organs of $\mathrm{CP}-/$ - mice [16]. Iron staining was blue in spleen, while the positive iron staining was not seen in the brain until it is enhanced with DAB. The data confirmed the fact that non-heme iron levels were very low in the brain, and also demonstrated that the staining of iron need to be enhanced with DAB and the positive staining is brown in color.

As for the different treatments with different fixative solutions, there were no obvious changes when the incubation time extended longer in Perl's solution (to 8hours). Figure 3 listed the recommended conditions in non-heme iron staining. It is noteworthy that these 4 kinds of fixative solutions have their own specific advantages in the maintenance of tissue structures and molecules, so optimal fixative solutions should be selected recombination with other considerations in experiments such as the conservation of antigen intensity in immunohistochemistry, interaction of fixative solutions with fat and sugar in tissues, the morphological changes of other organs in the same animals, and so on.

\begin{tabular}{|l|c|c|c|c|c|c|c|}
\hline Fixative solutions & Spleen & Midbrain & Cerebellum & Cpu & Cerebral Cortex & Corpus collosum & Choroid plexus \\
\hline $\mathbf{9 5} \%$ ethanol & DAB $(-), 1 \mathrm{~h}$ & $\mathrm{DAB}(+), 8 \mathrm{~h}$ & $\mathrm{DAB}(+), 8 \mathrm{~h}$ & $\mathrm{DAB}(+), 8 \mathrm{~h}$ & $\mathrm{DAB}(+), 8 \mathrm{~h}$ & $\mathrm{DAB}(+), 8 \mathrm{~h}$ & $\mathrm{DAB}(+), 8 \mathrm{~h}$ \\
\hline A mixture & $\mathrm{DAB}(-), 1 \mathrm{~h}$ & $\mathrm{DAB}(+), 8 \mathrm{~h}$ & $\mathrm{DAB}(+), 8 \mathrm{~h}$ & $\mathrm{DAB}(+), 8 \mathrm{~h}$ & $\mathrm{DAB}(+), 8 \mathrm{~h}$ & $\mathrm{DAB}(+), 8 \mathrm{~h}$ & $\mathrm{DAB}(+), 8 \mathrm{~h}$ \\
\hline Bouin's solution & $\mathrm{DAB}(-), 1 \mathrm{~h}$ & $\mathrm{DAB}(+), 8 \mathrm{~h}$ & $\mathrm{DAB}(+), 8 \mathrm{~h}$ & $\mathrm{DAB}(+), 8 \mathrm{~h}$ & $\mathrm{DAB}(+), 8 \mathrm{~h}$ & $\mathrm{DAB}(+), 8 \mathrm{~h}$ & $\mathrm{DAB}(+), 8 \mathrm{~h}$ \\
\hline $\mathbf{4 \%}$ polyformaldehyde & $\mathrm{DAB}(-), 1 \mathrm{~h}$ & $\mathrm{DAB}(+), 8 \mathrm{~h}$ & $\mathrm{DAB}(+), 8 \mathrm{~h}$ & $\mathrm{DAB}(+), 8 \mathrm{~h}$ & $\mathrm{DAB}(+), 8 \mathrm{~h}$ & $\mathrm{DAB}(+), 8 \mathrm{~h}$ & $\mathrm{DAB}(+), 8 \mathrm{~h}$ \\
\hline
\end{tabular}

Figure 3 : The summary diagram for recommended fixative solutions and DAB enhancement in non-heme iron staining. "A mixture" means a mixture of methanol: chloroform: acetic acid (volume ratio is 6:3:1), "DAB(-)" means non-heme staining without DAB enhancement, "DAB $(+)$ " means non-heme staining with DAB enhancement, " $1 \mathrm{~h}$ " or " $8 \mathrm{~h}$ " means the incubation time in Perl's staining solution is 1 hour or 8 hours, respectively.

In conclusion, DAB enhancement is needed in non-heme iron staining in the brain sections, and different fixative solutions have no significant effects on non-heme iron staining when the incubation time is long enough in Perl's solution. However, it still needs select the optimal fixative solution when it is not merely focusing on iron distribution and contents in studies.

\section{Perspectives}

We should know that the detection of iron distribution and contents is not merely limited to Perl's staining, more optional iron detection methods are developing in the brain, such as Magnetic mapping of iron at the nano scale level [17]. Nevertheless, Perl's staining has the superiority the other methods because this method is simple, accurate, reliable and reproducible. Although there are no obvious differences among different fixative solutions in our studies, it will provide important experimental foundations for selections of fixative solutions when other methods, such as immuno-fluorescence, in situ hybridization and single cell sequencing, were combined with Perl's staining, which will give more valuable information for future research.

\section{Acknowledgment}

This work was supported by the Hebei Provincial Natural Science Foundation of China (C2017205140).

\section{References}

1. Armitage AE, Drakesmith $H$ (2014) The battle for iron. Science 346(6215): 1299-1300.

2. Ke Y, Qian ZM (2007) Brain iron metabolism: neurobiology and neurochemistry. Prog Neurobiol 83(3): 149-173.

3. Hentze MW, Muckenthaler MU, Galy B, Camaschella C (2010) Two to tango: regulation of Mammalian iron metabolism. Cell 142(1): 24-38.

4. Lozoff B, Beard J, Connor J, Barbara F, Georgieff M, et al. (2006) Longlasting neural and behavioral effects of iron deficiency in infancy. Nutr Rev 64 (5 Pt 2): S34-S43.

5. Lozoff B, Jimenez E, Hagen J, Mollen E, Wolf AW (2000) Poorer behavioral and developmental outcome more than 10 years after treatment for iron deficiency in infancy. Pediatrics 105(4): E51.

6. Geng L, Duan X, Wang Y, Zhao Y, Gao G, et al. (2016) Quantum dotshemin: Preparation and application in the absorption of heme iron. Nanomedicine: Nanotechnology, biology and medicine 12(7): 17471755.

7. Guo X, Zheng H, Guo Y, Wang Y, Anderson GJ (2017) Nasal delivery of nanoliposome- encapsulated ferric ammonium citrate can increase the iron content of rat brain. J Nanobiotechnology 15(1): 42.

8. Smith MA, Harris PL, Sayre LM, Perry G (1997) Iron accumulation in Alzheimer disease is a source of redox-generated free radicals. Proc Natl Acad Sci U S A 94(18): 9866-9868.

9. Patel BN, Dunn RJ, Jeong SY, Zhu Q Julien JP (2002) Ceruloplasmin regulates iron levels in the CNS and prevents free radical injury. J Neurosci 22(15): 6578-6586. 
10. Asano Y (2012) Age-related accumulation of non-heme ferric and ferrous iron in mouse ovarian stroma visualized by sensitive non-heme iron histochemistry. J Histochem Cytochem 60(3): 229-242.

11. Jakeman A, Thompson T, McHattie J, Lehotay DC (2001) Sensitive method for non transferrin-bound iron quantification by graphite furnace atomic absorption spectrometry. Clin Biochem 34(1): 43-47.

12. Meguro R, Asano Y, Odagiri S, Li C, Shoumura K (2008) Cellular and subcellular localizations of nonheme ferric and ferrous iron in the rat brain: a light and electron microscopic study by the perfusion-Perls and -Turnbull methods. Arch Histol Cytol 71(4): 205-222.

13. Yu P, Chang YZ, Miao W, Wang SM, Cui R, et al. (2009) Effects of intracerebroventricular injection of iron dextran on the iron concentration and divalent metal transporter 1 expression in the caudate putamen and substantia nigra of rats. Anat Rec (Hoboken) 292(2): 225-233.

14. Lai D, Teng F, Hammad S, Werle J, Maas T, et al. (2018) Hepatic Smad7 overexpression causes severe iron overload in mice. Blood 131(5): 581585.

15. Muckenthaler MU, Rivella S, Hentze MW, Galy B (2017) A Red Carpet for Iron Metabolism. Cell 168(3): 344-361.

16. You LH, Li F, Wang L, Zhao SE, Wang SM, et al. (2015) Brain iron accumulation exacerbates the pathogenesis of MPTP-induced Parkinson's disease. Neuroscience 284: 234-246.

17. Blissett AR, Ollander B, Penn B, McTigue DM, Agarwal G (2017) Magnetic mapping of iron in rodent spleen. Nanomedicine 13(3): 977-986.
Creative Commons Attribution 4.0 International License

For possible submissions Click Here

\section{Submit Article}

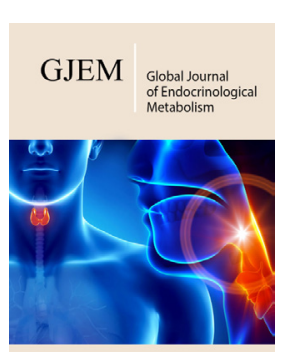

Global Journal of Endocrinological Metabolism

\section{Benefits of Publishing with us}

- High-level peer review and editorial services

- Freely accessible online immediately upon publication

- Authors retain the copyright to their work

- Licensing it under a Creative Commons license

- Visibility through different online platforms 